\title{
A transfer matrix model of the IEC 60318-4 ear simulator: application
}

\section{to the simulation of earplug insertion loss}

Final version 2019

Yu Luan ${ }^{1)}$, Franck Sgard ${ }^{2)}$, Simon Benacchio') ${ }^{1)}$ Hugues Nélisse $^{2)}$, Olivier Doutres ${ }^{1)}$

1) Department of Mechanical Engineering, École de Technologie Supérieure, 1100 rue

Notre-Dame Ouest, Montreal, Quebec H3C 1K3, Canada

2) IRSST, Direction Scientifique, 505 Boulevard de Maisonneuve Ouest, Montreal, Quebec H3A 3C2, Canada

\section{Summary}

The IEC 60318-4 ear simulator is used to measure the insertion loss (IL) of earplugs in the ear canal of an acoustical test fixture (ATF) and is designed to represent an average acoustic impedance (in a reference plane) of the human ear. The ear simulator is usually modeled using a lumped parameter model (LPM) which has frequency limitations and inadequately accounts for the thermo-viscous effects in the simulator. The simulator numerical models that can better deal with the thermo-viscous phenomena often lack essential geometric details. Most related studies also suffer from the lack of experimental validation of the models. Therefore, a transfer matrix (TM) model of the IEC 60318-4 simulator is proposed based on a direct assessment of its geometric dimensions. Such a model is of particular interest for designing artificial ear simulators. The variability in the simulator impedance due to the geometric uncertainties is quantified using the Monte Carlo method. The TM model is validated using i) a finite element (FE) model of the simulator and ii) impedance measurements with a sound intensity probe. It is found to better describe the simulator impedance above $3 \mathrm{kHz}$ compared to the LPM. The TM model is then coupled to a FE model of an occluded ATF ear canal to simulate the IL of an earplug in the frequency range $[100 \mathrm{~Hz}, 10 \mathrm{kHz}]$. In the model, the simulator is considered as a cylindrical cavity terminated by an equivalent tympanic impedance which is determined from the TM model to simulate the sound pressure measured at the real microphone position (not at the reference plane) in the ATF ear canal. The simulated IL is validated against i) that obtained with a complete FE model of the corresponding system and ii) measurements using an ATF. The TM model is shown to better agree with the simulator FE model than the LPM above $6 \mathrm{kHz}$ regarding the earplug IL simulated using this method.

A glossary summarizing the acronyms used in this paper is provided in Appendix B. 


\section{Introduction}

Acoustical test fixtures (ATFs) [1] can be used instead of human subjects to measure the sound attenuation of individual hearing protection devices, such as earplugs [2] since they can provide repeatable results and accommodate a large variety of test signals [3]. One of the indicators of the earplug attenuation is the insertion loss (IL), which corresponds to the difference between the sound pressure levels measured close to the position of the eardrum (also called tympanic membrane) with and without the earplug [2]. The ATF generally consists of an acoustically rigid artificial head and a cylindrical ear canal of constant circular cross-section [2]. The inner portion of this ear canal is normally included in an IEC 60318-4 occluded ear simulator (formerly known as IEC 711 ear simulator). The latter is fabricated using acoustic resonant elements tuned in a manner that the acoustic impedance at its entrance represents an average input impedance of the inner part of the human ear canal at a reference plane ${ }^{1}$ up to $10 \mathrm{kHz}[5,6]$. A microphone is placed at the output of the simulator where the recorded sound pressure would be as close as possible to that measured at the eardrum of an average human ear [4]. Such simulator contains the information about the average eardrum acoustic properties which may be beneficial for designing more realistic artificial ear simulators in the field of hearing protectors.

The IEC 60318-4 ear simulator was originally designed with the help of a lumped parameter model (LPM) which assimilates the simulator as an analogous electrical circuit whose parameters are associated with the acoustic properties of the simulator components $[7,8]$. A reliable analytical impedance model is of particular interest in terms of easily performing parametric studies to design artificial ear simulators. However, the LPM is commonly accepted to be limited to the low frequency range and often inadequately accounts for the thermo-viscous effects due to the sound propagation through the narrow regions of the simulator $[8,9]$.

Several numerical models of the IEC 60318-4 simulator based on the finite element (FE) or boundary element method have been proposed to better account for the thermo-viscous phenomena involved in the system $[7,8,10$, 11]. But most of the existing simulator numerical models $[10,11]$ were validated against experimental impedance data taken from international standards $[5,6]$ which were obtained using simultaneously an insert earphone and a probe microphone fixed in the human ear canal (e.g., $[4,12,13,14])$. They suffer from the lack of experimental validation of the models using the corresponding ear simulators. In addition, these studies either rely upon the adjusted component dimensions to acquire the closest impedance simulation results to the measurement data on human subjects or lack essential details associated with the simulator geometric parameters.

To the authors' knowledge, the only study on the modeling of an ear simulator based on a direct determination of its geometric inputs was conducted by Bravo et al. [9, 15]. It proposed a FE model to calculate the transfer impedance of an IEC 60318-1 simulator [16] whose geometry was constructed using an X-ray inspection system. However, the uncertainties related to the simulator geometry were only considered using the maximal and minimal values of two particular component dimensions, which seems not sufficient to represent the large variability in

\footnotetext{
${ }^{1}$ Due to the difficulty of directly assessing the eardrum impedance, measurements of the acoustic impedance of the human ear are practically performed at a reference plane in the ear canal between the canal entrance and eardrum where earplugs (or ear moulds) normally end [4].
} 
the simulator impedance that could be induced by different combinations of the geometric inputs. Moreover, this kind of simulator is designed for supra-aural or supra-cocha devices and cannot be adopted to evaluate the attenuation of earplugs $[16,17]$. A comprehensive review about different types of ear simulators can be found in $[17]$.

Besides the direct impedance simulation of the IEC 60318-4 simulator, it is relevant to assess how the simulator impedance model affects the simulated IL of earplugs inserted in an ATF ear canal. A 2D axisymmetric FE model of the corresponding system has been proposed in a few studies to precisely capture the vibroacoustic behavior of a silicone earplug $[18,19]$. In the model, the ear simulator was considered as a cylindrical cavity terminated by an equivalent tympanic impedance (TI) in order to simulate the sound pressure measured at the real microphone position (not at the reference plane) in the ATF ear canal. The equivalent TI was determined by eliminating the components associated with the ear canal portion from the LPM of the simulator as originally proposed in [7]. But there is still doubt with regard to the validity of this traditional lumped TI model since it seems not mathematically rigorous to represent the TI by extracting directly certain elements from the simulator equivalent circuit. Several published investigations have adopted the reduced impedance (RI) method [20] to retrieve the eardrum impedance from the acoustic impedance measured at a reference plane in the human ear canal using a chain matrix of the canal considered as a stepped duct (e.g., [21, 17, 22]). It proves to be a reliable method but has not yet been applied to calculate the equivalent TI of the IEC 60318-4 simulator. In addition, one can wonder whether it is valid to consider such a simulator as a cavity with a locally reacting terminal impedance condition for simulating the sound pressure in an ATF ear canal occluded by an earplug.

To address the above-mentioned lacks in the literature, the main objective of this work is to propose a reliable analytical impedance model of the IEC 60318-4 simulator and apply it to the IL simulation of earplugs in an ATF ear canal. This objective is achieved via a three-step methodology detailed hereafter. Firstly, the geometry of a commercial model of the IEC 60318-4 occluded ear simulator is identified from a micro-Computed Tomography (micro-CT) scan. A transfer matrix (TM) model of the simulator is proposed based on the component dimensions assessed from CT scan images and compared to the LPM. The variability in the model output (simulator impedance) due to the uncertainties on the geometric inputs is quantified using the Monte Carlo method. The proposed model is validated using i) a FE model of the scanned simulator which includes full and detailed modeling of the thermo-viscous phenomena involved in the system and ii) measurements carried out with a sound intensity probe. Secondly, the equivalent TI is determined from the TM model and LPM using the RI method and compared to that obtained from the traditional lumped TI model. Thirdly, TI results are used as a boundary condition in a FE model of an occluded ATF ear canal to simulate the IL of a silicone earplug. A typical silicone earplug is chosen because it has already been studied in the literature and its FE model has been shown to provide satisfactory attenuation simulation results compared to experimental data [18, 19]. The simulated IL based on the TM model is validated against i) that obtained with a complete FE model of the corresponding system and ii) measurements using an ATF. The validity of considering the ear simulator as a cylindrical cavity 
Table 1: Component geometric dimensions of the IEC 60318-4 ear simulator

\begin{tabular}{ccc}
\hline Notation & Parameter & $\begin{array}{c}\text { Average } \pm \\
\text { standard deviation }\end{array}$ \\
\hline$R_{0}$ & Radius of the main cavity & $3.77 \pm 0.03 \mathrm{~mm}$ \\
$L_{0}$ & Total length of the main cavity & $12.56 \pm 0.29 \mathrm{~mm}$ \\
$L_{1}$ & Length: $1^{\text {st }}$ section of the main cavity & $3.12 \pm 0.10 \mathrm{~mm}$ \\
$L_{3}$ & Length: $2^{\text {nd }}$ section of the main cavity & $4.75 \pm 0.04 \mathrm{~mm}$ \\
$L_{5}$ & Length: $3^{\text {rd }}$ section of the main cavity & $4.69 \pm 0.14 \mathrm{~mm}$ \\
$a_{2}$ & Length of the rectangular slit & $2.53 \pm 0.06 \mathrm{~mm}$ \\
$b_{2}$ & Width of the rectangular slit & $2.35 \pm 0.03 \mathrm{~mm}$ \\
$h_{2}$ & Thickness of the rectangular slit & $0.16 \pm 0.06 \mathrm{~mm}$ \\
$r_{2}$ & Inner radius of the $1^{\text {st }}$ annular cavity & $6.30 \pm 0.06 \mathrm{~mm}$ \\
$R_{2}$ & Outer radius of the $1^{\text {st }}$ annular cavity & $9.01 \pm 0.03 \mathrm{~mm}$ \\
$d_{1}$ & Thickness of the $1^{\text {st }}$ annular cavity & $1.91 \pm 0.08 \mathrm{~mm}$ \\
$r_{4}$ & Outer radius of the annular slit (Inner radius of the $2^{\text {nd }}$ annular cavity) & $4.66 \pm 0.04 \mathrm{~mm}$ \\
$\alpha_{2}$ & Angle of each part of the annular slit & $95.33 \pm 1.09^{\circ}$ \\
$h_{4}$ & Thickness of the annular slit & $0.05 \pm 0.02 \mathrm{~mm}$ \\
$R_{4}$ & Outer radius of the $2^{\text {nd }}$ annular cavity & $9.01 \pm 0.03 \mathrm{~mm}$ \\
$d_{2}$ & Equivalent thickness of the $2^{\text {nd } \text { annular cavity }}$ & $1.40 \pm 0.11 \mathrm{~mm}$ \\
\hline
\end{tabular}

terminated by an equivalent TI is verified and the effects of the TI models on the IL simulation are discussed.

\section{Modeling of the IEC 60318-4 ear simulator}

This section presents different modeling strategies to simulate the input impedance of the IEC 60318-4 simulator and is organized as follows. The simulator geometry and key dimensions determined from a CT scan are given in Section 2.1. Section 2.2 recalls the principles of the LPM of the ear simulator. Section 2.3 provides the details of a TM model of the simulator. To validate the TM model, a FE model of the scanned simulator is constructed and described in Section 2.4. Note that in this paper the pressure-volume velocity analogy is adopted so that the term "impedance" refers to acoustic impedance.

\subsection{Geometry}

The IEC 60318-4 ear simulator studied in this work is the G.R.A.S. RA0045 (G.R.A.S. Sound \& Vibration AS, Denmark) which complies with the requirements of IEC 60318-4 [5]. The geometry of the simulator is identified from a micro-CT scan with a $15.4 \mu \mathrm{m}$ isotropic resolution (XT H 225 micro-CT X-Ray Scanner, Nikon Metrology, United States) (see Fig.1). It is made of hard nonporous material and consists of a cylindrical tube of constant circular cross-section (main cavity) (component A) attached to two annular side volumes (components C, E) via two narrow slits (components B, D). The main cavity of the simulator approximates the human ear canal portion and is divided into three sections by the narrow slits. The latter include thin air layers in which thermo-viscous boundary effects cannot be neglected, mimicking the energy losses at the human eardrum and those in the middle and inner ears. Each narrow slit and the corresponding side cavity constitute a Helmholtz resonator.

\footnotetext{
${ }^{2}$ The second side cavity is simplified to a pure annular form in the TM model since it has been proved that only the volume of the cavity affects the simulated impedance of the ear simulator. An equivalent thickness that preserves the same volume of this cavity is provided in Table 1.
} 


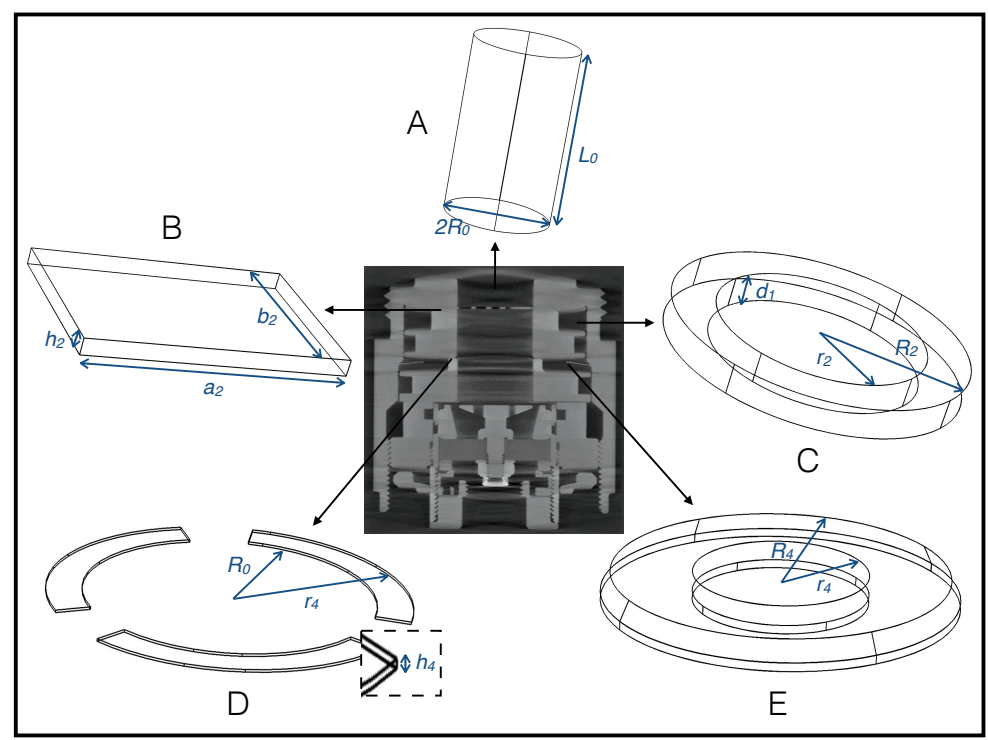

Figure 1: Geometry of the IEC 60318-4 ear simulator identified from the CT scan (see Table 1 for geometric dimensions)

The first slit is of parallelepipedic shape (component B) while the second one consists of three identical annular parts (component D). The second side cavity ${ }^{2}$ (component E) comprises a conical part containing a ring-shaped sheet metal. The IEC 60318-4 simulator is generally terminated by a recording microphone at its output (eardrum position). In this study, it is replaced by a rigid boundary (infinite terminal impedance) for simplicity because the impedance of the microphone is very high compared to that of the system in front [5].

The dimensions of the identified acoustic elements (i.e., main cavity, narrow slits and side cavities) have been assessed by measurements on micro-CT scan images using VGSTUDIO (Volume Graphics, Germany). Different measurements were performed by three members of the research team to obtain the average and standard deviation for each geometric dimension of interest (see Table $1^{2}$ ).

\subsection{Lumped parameter model}

If the wavelength is sufficiently large compared to the dimensions of the acoustic components in the IEC 60318-4 simulator, these components can be modeled as lumped acoustic elements. In this case, the simulator can be considered as an analogous electrical circuit or lumped parameter model (LPM).

Figure 2 presents the LPM of the studied ear simulator built according to [8], the principles of which are recalled as follows. In this model, each section of the simulator main cavity is represented by an LC circuit where the electrical inductance and capacitance correspond respectively to the acoustic mass and compliance of the cavity portion. The latter can be derived from [8]:

$$
\left\{\begin{array}{l}
m_{a, m}=\frac{\rho_{0} L_{m}}{S_{0}} \\
c_{a, m}=\frac{V_{m}}{\rho_{0} c_{0}^{2}}
\end{array}\right.
$$




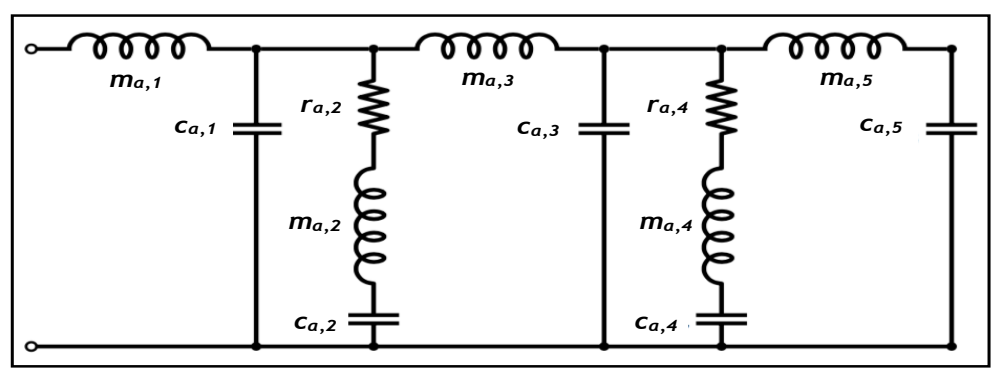

Figure 2: LPM of the IEC 60318-4 ear simulator

where $\mathrm{m}=1,3$ and 5 correspond to each section of the simulator main cavity, $L_{m}$ denotes the length of each section, $V_{m}$ the volume of the section correspondingly and $S_{0}$ is the cross-section area of the simulator main cavity equal to $\pi R_{0}^{2} \cdot \rho_{0}$ is the density of air and $c_{0}$ is the speed of sound in air. Parameters of the thermo-viscous fluid adopted in the models are derived from the standard atmospheric pressure and normal room temperature, and are specified in Table 2. In the same way, each RLC circuit in the LPM corresponds to a Helmholtz resonator in which the electrical resistance and inductance match the acoustic resistance and mass of the resonator's neck (narrow slit), and the electrical capacitance matches the acoustic compliance of the resonator's cavity. Thus, the input impedance of the Helmholtz resonator can be calculated by [8]:

$$
Z_{H R, n}=r_{a, n}+j \omega m_{a, n}+\frac{1}{j \omega c_{a, n}}
$$

$\mathrm{n}=2$ and 4 correspond respectively to the first and second Helmholtz resonators. $c_{a, n}$ can be determined as Eq.(1) and $r_{a, n}, m_{a, n}$ are obtained from the simulator geometric properties by [8]:

$$
\left\{\begin{array}{l}
r_{a, n}=\frac{12 \mu a_{n}}{b_{n} h_{n}^{3}} \\
m_{a, n}=\frac{6 \rho_{0} a_{n}}{5 S_{s l i t, n}}
\end{array}\right.
$$

where $a_{n}, b_{n}$ and $h_{n}$ are respectively the length, width and thickness of the narrow slit, $S_{\text {slit,n }}$ corresponds to the cross-section area of the rectangular or annular slit, and $\mu$ denotes the shear dynamic viscosity of air.

\subsection{Transfer matrix model}

When considering each acoustic element in the IEC 60318-4 simulator as a two-port system, the acoustic pressure and volume velocities at the inlet and outlet of the element can be related using a transfer matrix. For each section of the simulator main cavity, one has:

$$
\boldsymbol{T}_{\mathbf{m}}=\left[\begin{array}{cc}
\cos \left(k_{0} L_{m}\right) & j Z_{1} \sin \left(k_{0} L_{m}\right) \\
j \sin \left(k_{0} L_{m}\right) / Z_{1} & \cos \left(k_{0} L_{m}\right)
\end{array}\right]
$$


Table 2: Parameters of the thermo-viscous fluid

\begin{tabular}{lll}
\hline Parameter & Value & Unit \\
\hline Static pressure $P_{0}$ & $1.01 \times 10^{5}$ & $\mathrm{~Pa}$ \\
Temperature $T_{0}$ & 293.15 & $\mathrm{~K}$ \\
Density $\rho_{0}$ & 1.20 & $\mathrm{~kg} / \mathrm{m}^{3}$ \\
Speed of sound $c_{0}$ & 343.90 & $\mathrm{~m} / \mathrm{s}$ \\
Shear dynamic viscos- & $1.82 \times 10^{-5}$ & $\mathrm{~Pa} \cdot \mathrm{s}$ \\
ity $\mu$ & & \\
Thermal conductivity $\lambda$ & $24.80 \times 10^{-3}$ & $\mathrm{~W} /(\mathrm{mK})$ \\
Ratio of specific heats $\gamma$ & 1.40 & - \\
Specific heat coefficient & $1.00 \times 10^{3}$ & $\mathrm{~J} /(\mathrm{kgK})$ \\
at constant pressure per & & \\
unit of mass $C_{p}$ & & \\
\hline
\end{tabular}

$\mathrm{m}=1,3$ and 5 correspond respectively to each section of the main cavity, $k_{0}$ denotes the wavenumber and $Z_{1}$ denotes the characteristic impedance of the main cavity equal to $\rho_{0} c_{0} / S_{0}$. For each Helmholtz resonator:

$$
\boldsymbol{T}_{\mathbf{n}}=\left[\begin{array}{cc}
1 & 0 \\
1 / Z_{H R, n} & 1
\end{array}\right]
$$

$\mathrm{n}=2$ and 4 correspond to the first and second Helmholtz resonators. $Z_{H R, n}$ corresponds to the impedance at the input of the first or second resonator. The input impedance of the rectangular and annular slits is derived using the low reduced frequency (LRF) model in which the thermal and viscous energy losses in the fluid are taken into account in a homogeneous way using the complex wavenumber and characteristic impedance [23, 24]. The detailed calculation process of $Z_{H R, n}$ based on the LRF model is provided in Appendix A.

The global transfer matrix of the IEC 60318-4 simulator can be obtained by assembling the one related to each element of the system:

$$
\boldsymbol{T}^{\boldsymbol{E S}}=\left[\begin{array}{cc}
T_{11}^{E S} & T_{12}^{E S} \\
T_{21}^{E S} & T_{22}^{E S}
\end{array}\right]=\boldsymbol{T}_{\mathbf{1}} \boldsymbol{T}_{\mathbf{2}} \boldsymbol{T}_{\mathbf{3}} \boldsymbol{T}_{\mathbf{4}} \boldsymbol{T}_{\mathbf{5}}
$$

The reflection coefficient of the simulator in the case of an infinite terminal impedance can be determined by:

$$
R_{s}=\frac{T_{11}^{E S}-T_{21}^{E S} Z_{1}}{T_{11}^{E S}+T_{21}^{E S} Z_{1}}
$$

The input impedance of the ear simulator is derived from Eq.(7):

$$
Z_{s}=Z_{1} \frac{1+R_{s}}{1-R_{s}}
$$




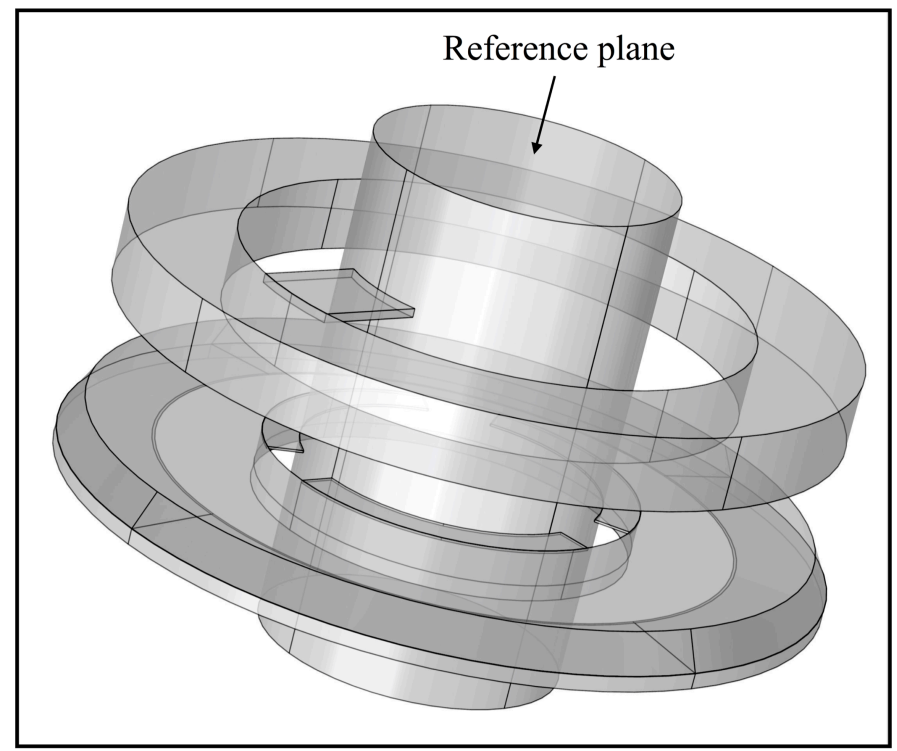

Figure 3: Geometry used in the FE model of the IEC 60318-4 ear simulator

\subsection{Finite element model}

To validate the TM model, a detailed FE model of the G.R.A.S. RA0045 simulator is constructed in COMSOL Multiphysics (v.5.3a COMSOL®, Sweden) following the modeling strategy of [11] based on its scanned geometry (see Fig.1). The entrance of the simulator (reference plane) is marked in Fig.3. The sound propagation in the main cavity and side volumes of the simulator is governed by Helmholtz equation in which energy losses are not considered. This choice is made since the thermo-viscous effects in these regions are negligible and those around the ring-shaped sheet metal in the second side cavity have been proved to have very little influence on the simulated impedance of the simulator. These domains are meshed using quadratic 10-noded tetrahedral elements based on a meshing criterion of at least 6 elements per wavelength. On the other hand, thermo-viscous acoustic domains are adopted for the narrow slits where the sound propagation is governed by linearized Navier-Stokes equations with viscous and heat conduction effects taken into account [25]. These domains are meshed using a dense element distribution in the normal direction along the horizontal walls of the slits to accurately resolve the acoustic boundary layers within which thermal and viscous dissipation is significant. The sound pressure, particle velocity and temperature are computed at each node of the domains. The coupled problem is solved using the built-in Acoustics/Thermoviscous Acoustics Multiphysics boundary coupling condition in COMSOL Multiphysics. An acoustically rigid condition is introduced to the outlet of the simulator and a displacement excitation of $10^{-5} \mathrm{~m}$ is imposed at the reference plane. The input impedance of the simulator is calculated using the sound pressure $p$ and particle velocity $\vec{v}$ determined at each node of the simulator entrance:

$$
Z_{s}=\frac{1}{S_{0}} \frac{\int_{S_{0}} p \mathrm{~d} S}{\int_{S_{0}} \vec{v} \cdot \vec{n} \mathrm{~d} S}
$$




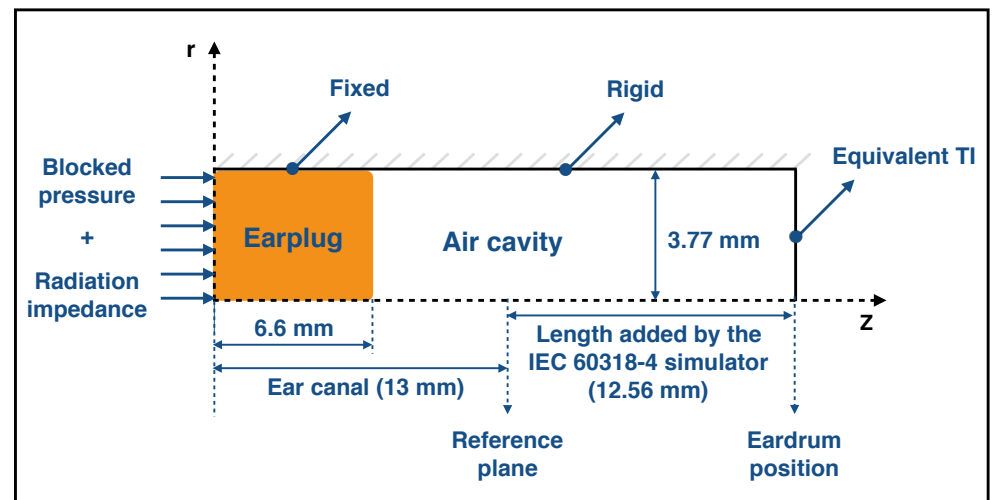

Figure 4: Schematic representation of the FE model to simulate the IL of a silicone earplug (Adapted from [19])

\section{Modeling of the open and occluded ATF ear canals}

\subsection{IL of the earplug}

The IL of a $6.6 \mathrm{~mm}$ long silicone custom-molded earplug inserted into the ear canal of an ATF is simulated using a 2D axisymmetric FE model in COMSOL Multiphysics (see Fig.4) following the modeling strategy of [18]. The ATF ear canal consists of a rigid-walled cylinder of constant circular cross-section terminated by an IEC 60318-4 simulator. In the model, the simulator is considered as a cylindrical air cavity terminated by an equivalent TI (see Section 3.2) in order to simulate the sound pressure at the eardrum position rather than that at the reference plane. The elastic isotropic mechanical properties of the silicone earplug adopted in the simulation are assessed using a quasi-static mechanical analyzer: density $\left(1500 \mathrm{~kg} \cdot \mathrm{m}^{-3}\right)$, Young's modulus (1.7 MPa), Poisson's ratio (0.48) and isotropic loss factor (0.18). A blocked pressure of $2 P a$ induced by a normal incident plane wave is imposed at $z=0$. In the specific case of the open ear, a normal acoustic particle acceleration condition is introduced at $z=0$ which depends simultaneously on the blocked pressure and the radiation impedance of a baffled circular piston to account for the interaction with the external fluid [26]. The calculation of the earplug IL requires the simulation of the sound pressure at the eardrum position in the open ear canal and that in the ear canal occluded by the earplug:

$$
I L=20 \log _{10}\left(\left|p_{\text {open }}\right|\right)-20 \log _{10}\left(\left|p_{\text {occluded }}\right|\right)
$$

Additionally, the earplug IL simulated with a complete FE model of the corresponding system (see Fig.5) which uses the detailed FE model of the IEC 60318-4 simulator (see Fig.3) is taken as the reference. The same modeling process as the 2D axisymmetric FE model is followed and the sound pressure at the output of the ear simulator (eardrum position) is simulated in order to calculate the earplug IL using Eq.(10). 


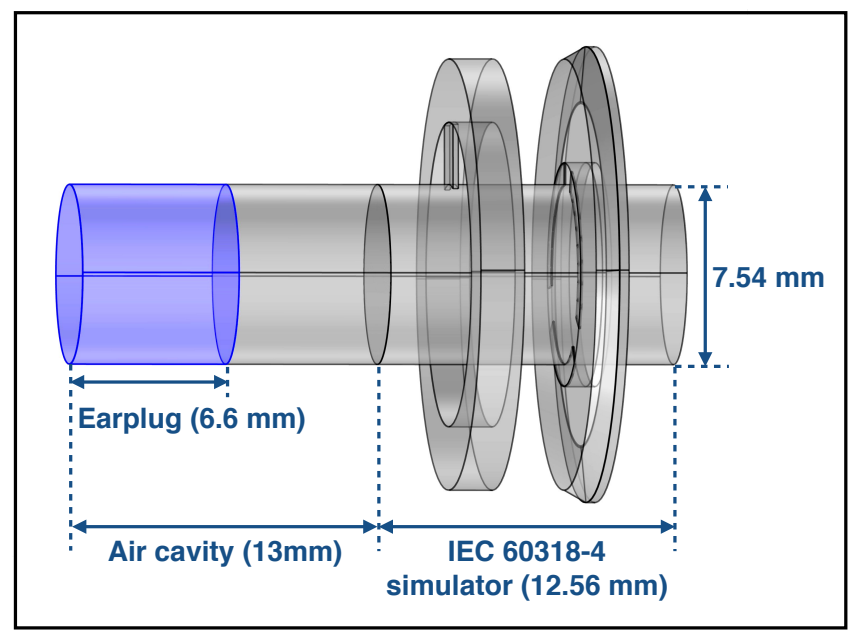

Figure 5: Geometry used in the complete FE model of an occluded ATF ear canal

\subsection{Equivalent TI}

A traditional way to obtain the equivalent TI of the IEC 60318-4 simulator is to extract the two RLC circuits in parallel from the LPM of the simulator (see Fig.2) which correspond to the Helmholtz resonators [18]. In this study, another approach based on the RI method is adopted to determine the TI from the input impedance of the simulator. Considering the entire ear simulator as a two-port system, the acoustic pressure and volume velocities at the reference plane $\left(p_{r p}, u_{r p}\right)$ and eardrum position $\left(p_{e p}, u_{e p}\right)$ can be related by a transfer matrix $[21,17,22]:$

$$
\left(\begin{array}{l}
p_{r p} \\
u_{r p}
\end{array}\right)=\left[\begin{array}{ll}
T_{11} & T_{12} \\
T_{21} & T_{22}
\end{array}\right]\left(\begin{array}{l}
p_{e p} \\
u_{e p}
\end{array}\right)
$$

with $T_{11}=\cos \left(k_{0} L_{0}\right), T_{12}=j Z_{1} \sin \left(k_{0} L_{0}\right), T_{21}=j \sin \left(k_{0} L_{0}\right) / Z_{1}$ and $T_{22}=\cos \left(k_{0} L_{0}\right) . L_{0}$ is the length between the reference plane and microphone position (eardrum position) of the simulator (see Fig.4). The equivalent TI is then given by:

$$
Z_{e p}=\frac{T_{22} Z_{r p}-T_{12}}{T_{11}-T_{21} Z_{r p}}
$$

where $Z_{r p}=p_{r p} / u_{r p}$ denotes the impedance at the reference plane. This method allows for calculating the TI once the input impedance of the simulator and its length are provided.

Specifically in this work, one is interested in three equivalent TI models based on the analytical impedance models of the IEC 60318-4 simulator: model 1 corresponds to the TI derived from the TM model of the simulator using the RI method, model 2 and model 3 are related to the TI obtained from the LPM of the simulator respectively with the RI method and in the traditional way using the RLC circuits in parallel. 


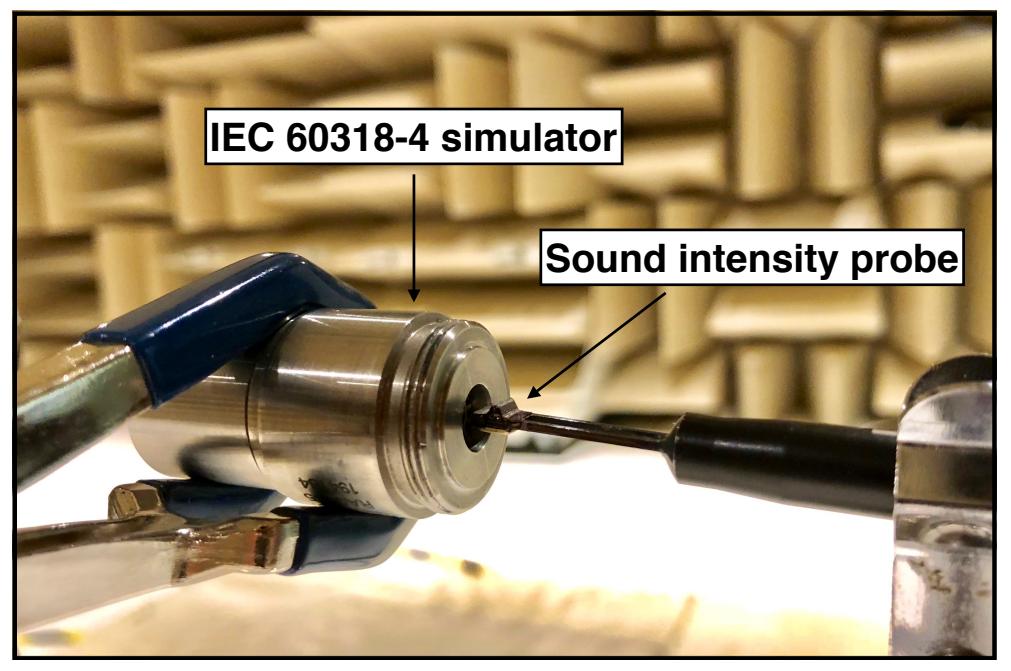

Figure 6: IEC 60318-4 ear simulator input impedance measurements using a sound intensity probe

\section{Experimental measurements}

\subsection{Measurements of the IEC 60318-4 ear simulator input impedance}

In order to validate the proposed TM model, the simulator impedance was measured using a sound intensity probe (PU Match-PTN, Microflown Technologies, Netherlands) which allows for determining the acoustic pressure and particle velocity in the frequency range from $100 \mathrm{~Hz}$ to $10 \mathrm{kHz}$ (see Fig.6). The system was excited acoustically in a semi-anechoic chamber with a white noise of $97 \mathrm{~dB}$ in overall sound pressure level generated by a loudspeaker (model K162 SN, dB Technologies, Italy). The center of the speaker was located at the same height (about 1 $\mathrm{m}$ ) as the center of the simulator and at a distance of about $2 \mathrm{~m}$. The floor around the simulator was covered with sound-absorbing foam to minimize ground reflections. The dust protector at the inlet of the simulator was removed and the intensity probe was placed as close as possible to the entrance of the simulator in order to precisely calculate the input impedance of the latter using the experimental data. Four successive measurements were performed on three G.R.A.S. RA0045 simulators of different serial numbers to account for the possible variability associated with the probe position and orientation.

\subsection{Measurements of the earplug IL using an ATF}

The IL of the silicone earplug was assessed at normal room temperature using an ATF (G.R.A.S. 45CB, G.R.A.S. Sound \& Vibration AS, Denmark) without pinna simulators which comprises a rigid-walled cylindrical ear canal terminated by an IEC 60318-4 simulator. The system was excited by a white noise of around $110 \mathrm{~dB}$ in overall sound pressure level in a reverberant room (diffuse field). The sound pressure levels in the open and occluded ear canals were measured using the recording microphone in the ear simulator (G.R.A.S. 40AG, G.R.A.S. Sound \& Vibration AS, Denmark). A reference microphone (MPA231, BSWA Technology Co., China) was positioned at about $1 \mathrm{~m}$ from the ear canal entrance in order to obtain the corresponding transfer functions between the two microphones which are used to calculate the earplug IL. The measurements were repeated three times to 


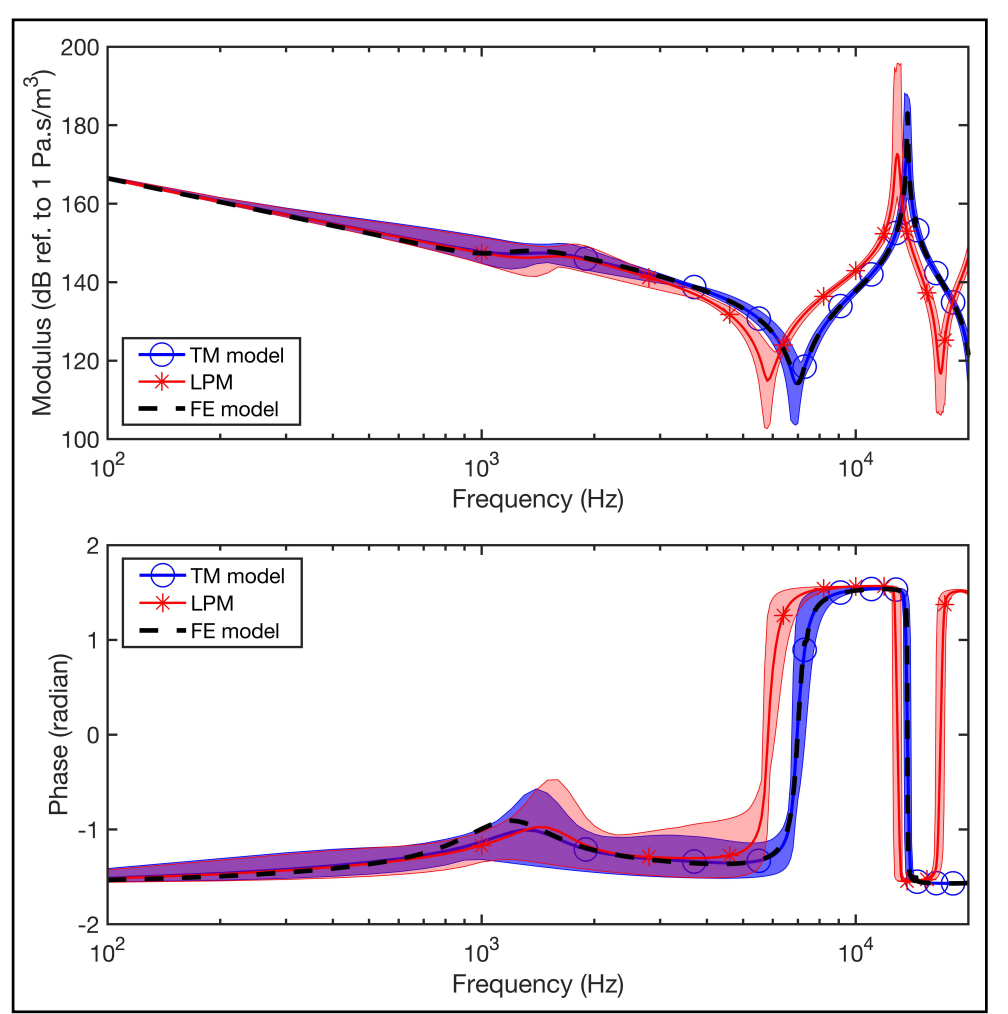

Figure 7: Input impedance of the IEC 60318-4 ear simulator obtained using different models: TM model (blue circles), LPM (red asterisks), FE model (dashed black). (top) modulus; (bottom) phase. Averaged simulation result together with the maximal and minimal values are plotted for each analytical model

evaluate the variability related to the mounting conditions. The details of the earplug IL measurements can be found in [19].

\section{Results and discussion}

\subsection{Input impedance of the IEC 60318-4 ear simulator}

Figure 7 illustrates the input impedance of the IEC 60318-4 ear simulator obtained from different models: the blue zone (circles) corresponds to the TM model, the red zone (asterisks) corresponds to the LPM and the dashed black line is related to the FE model of the simulator. For the analytical models, the uncertainty propagation of the simulator geometric dimensions is taken into account using the Monte Carlo method. 1000 evaluations have been run for each model in which a uniform distribution is chosen for any dimension concerned based on the corresponding average and standard deviation in Table 1. A convergence study has shown that this number of evaluations is sufficient to capture the variability in the simulator impedance (or equivalent TI) induced by its geometric uncertainties. The FE model is based on the mean component dimensions of the simulator. Results are presented in the frequency range from $100 \mathrm{~Hz}$ to $20 \mathrm{kHz}$ as all the relevant studies $[7,8,10,11]$ in order to exhibit the first resonance of the simulator main cavity around $13.5 \mathrm{kHz}$. In the following, results are displayed in the working frequency range of the ear simulator $(100 \mathrm{~Hz}-10 \mathrm{kHz})$ beyond which it does not necessarily represent 


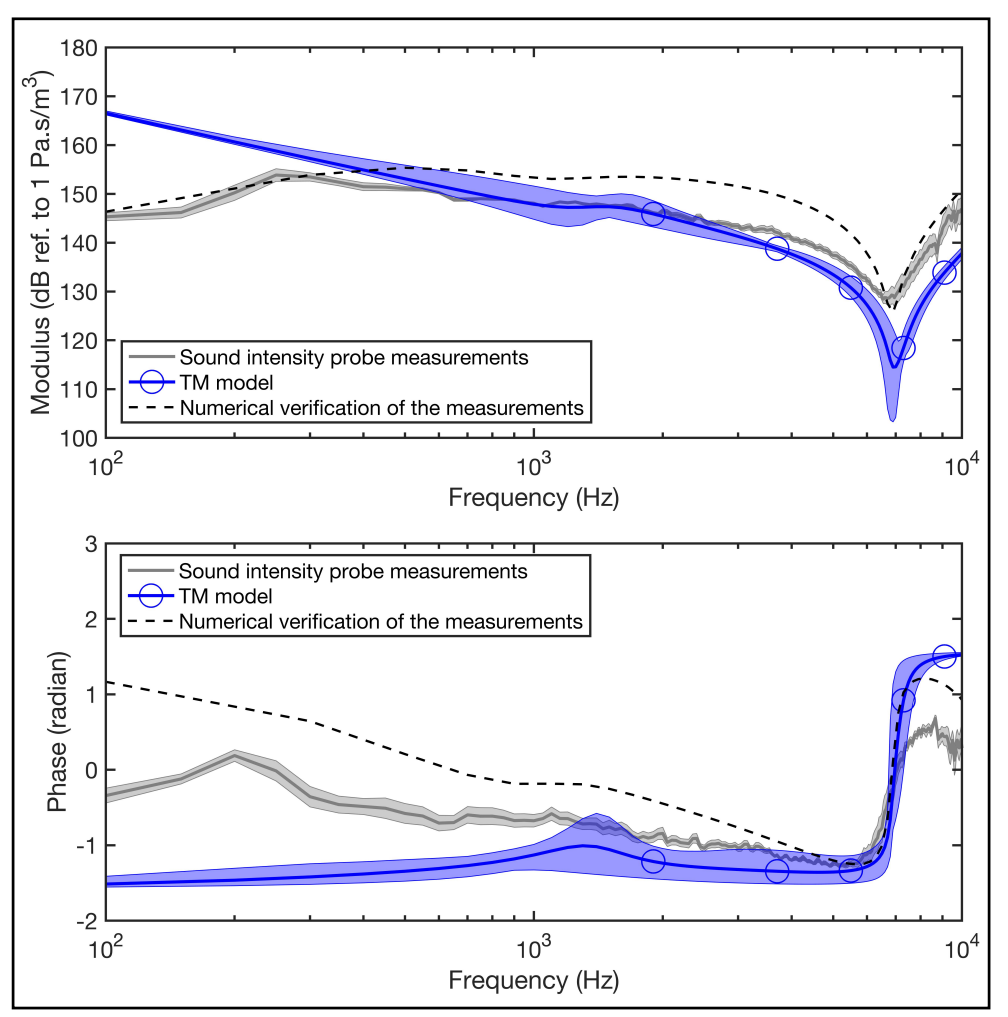

Figure 8: Measurement and simulation results of the IEC 60318-4 ear simulator input impedance: sound intensity probe measurements (average \pm standard deviation, grey), TM model (blue circles). Numerical verification result of the measurements (dashed black) is also presented. (top) modulus; (bottom) phase. Averaged simulation result together with the maximal and minimal values are plotted for the TM model

the acoustic impedance of the human ear [5]. The figure shows that all the models are able to provide similar impedance simulation results up to nearly $3 \mathrm{kHz}$. A frequency offset is observed from the LPM at medium and high frequencies (over $3 \mathrm{kHz}$ ) compared to the other models. In this frequency range, the LPM fails to precisely describe the behavior of the IEC 60318-4 simulator. This is consistent with previous findings of [9, 15] which used the LPM to calculate the acoustic impedance of an IEC 60318-1 simulator. Additional results not presented in the paper have shown that the LPM of the simulator main cavity does not satisfy the low frequency approximation, which is the reason for the observed frequency offset. The TM model is found in perfect agreement with the FE model of the simulator in the whole frequency range of interest, which proves the validity of the former. It is necessary to mention that the Helmholtz resonators of the IEC 60318-4 simulator have resonant frequencies at about $1400 \mathrm{~Hz}$ and $3800 \mathrm{~Hz}$, covering the behavior of the system in the mid-frequency range. The simulated simulator impedance (especially phase values) is shown to be very sensitive to the resonator geometric parameters in the associated frequency ranges. Around $1500 \mathrm{~Hz}$, the TM model is found to be slightly closer to the FE model than the LPM. This is probably due to the fact that the impedance of the first resonator's neck (rectangular narrow slit) is better captured by the LRF model than the LPM.

Figure 8 displays the measurement results using the sound intensity probe (grey zone) together with the simulator input impedance calculated by the TM model. Even though there do exist some discrepancies in certain frequency bands, a satisfactory agreement is seen between the simulation and measurements of the 


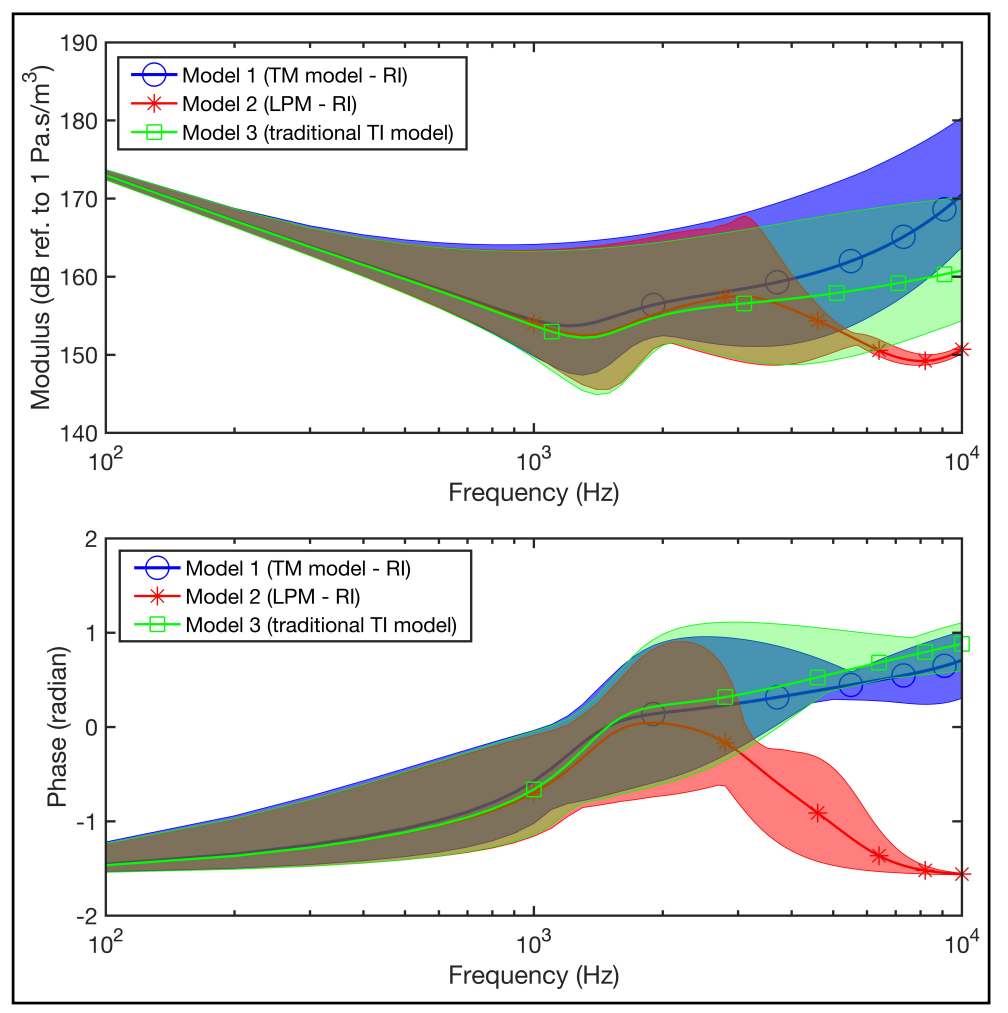

Figure 9: Different equivalent TI models of the IEC 60318-4 ear simulator: model 1 (blue circles), model 2 (red asterisks), model 3 (green squares). (top) modulus; (bottom) phase. Averaged simulation result together with the maximal and minimal values are plotted for each TI model

simulator impedance, which further gives confidence in the TM model of the simulator in the frequency range from $100 \mathrm{~Hz}$ to $10 \mathrm{kHz}$. The differences observed between the model and sound intensity probe measurements (mainly below $700 \mathrm{~Hz}$ and above $7 \mathrm{kHz}$ ) are partly associated with the sound diffraction of the probe positioned in front of the simulator all along the experiments as shown in Fig.8 (dashed black line). This curve has been obtained by accounting for the scattering from the sound intensity probe in the FE model of the ear simulator (see Fig.3). Note that in the simulation, a simplified shape of the probe has been considered and the probe sensor has been positioned approximately at the same place as in the experiments. Another possible reason is that the sound intensity probe was difficult to be placed right at the input surface of the simulator when performing the measurements and the interaction with the external sound field is not taken into account in the TM model.

\subsection{Equivalent TI}

Figure 9 illustrates different equivalent TI models of the IEC 60318-4 simulator accounting for its dimension uncertainties: the blue zone (circles) corresponds to model 1 (TM model - RI), the red zone (asterisks) corresponds to model 2 (LPM - RI) and the green zone (squares) corresponds to model 3 (traditional TI model). A wide variation due to the simulator geometric uncertainties is exhibited in the calculated TI at frequencies over 1 $\mathrm{kHz}$. Below $3 \mathrm{kHz}$, all the models provide similar TI simulation results which however become rather different at higher frequencies. Particularly, the obvious differences between model 2 (LPM - RI) and the first TI model (TM 


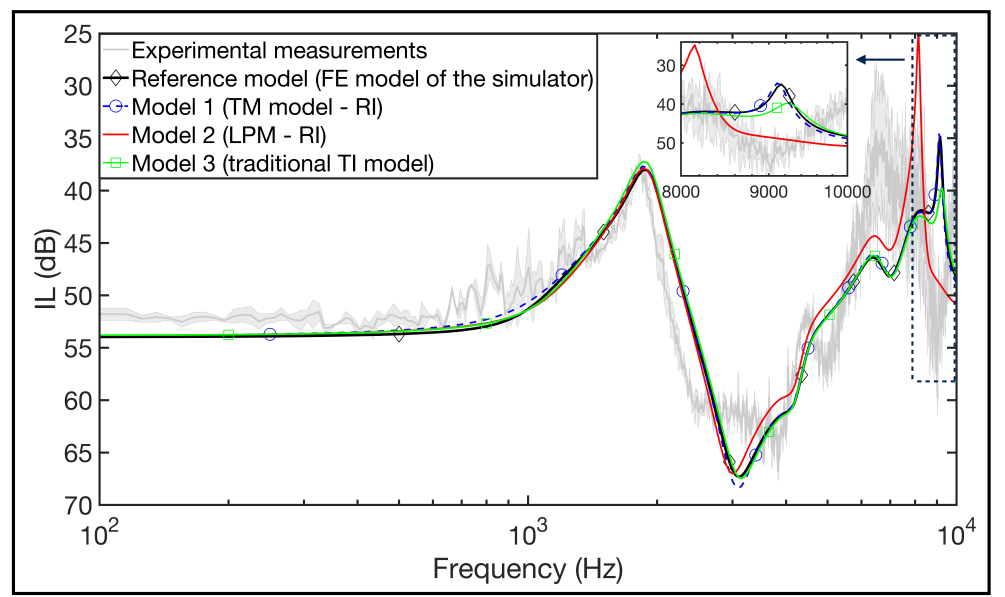

Figure 10: IL of the silicone earplug simulated using different equivalent TI models: model 1 (dashed blue circles), model 2 (red), model 3 (green squares). Experimental data (average \pm standard deviation, grey) and simulation result using the FE model of the ear simulator (black diamonds) are also presented

model - RI) over $3 \mathrm{kHz}$ could originate from the inconsistencies already seen in Fig.7 between the LPM and TM model of the simulator. These inconsistencies are due to the fact that the sound propagation in the simulator main cavity is better captured by the TM model than the LPM. Furthermore, model 3 (traditional TI model) even though not mathematically rigorous, seems not far from model 1 (TM model - RI) up to $7 \mathrm{kHz}$.

\subsection{IL of the earplug}

Figure 10 presents the silicone earplug IL simulated using the various TI models: the dashed blue curve (circles) corresponds to model 1 (TM model - RI), the red curve corresponds to model 2 (LPM - RI) and the green line (squares) is related to model 3 (traditional TI model). In addition, the earplug IL calculated using the FE model of the ear simulator (see Fig.5) is displayed as the reference (black curve with diamonds), together with the measurement results using the ATF (grey zone). All the models are based on the mean component dimensions of the simulator (see Table 1). From Fig.10, no remarkable effect of the TI models on the earplug IL simulation is observed up to $6 \mathrm{kHz}$. In this frequency range, the system is mainly controlled by the first resonance of the earplug around $1.9 \mathrm{kHz}$ which corresponds to a rigid body mode of the earplug with elastic boundary conditions [18, 19]. In accordance with Fig.7, model 1 (TM model - RI) compares well with the reference model (FE model of the simulator) in the whole frequency range of interest with regard to the simulated IL. This finding confirms that considering the IEC 60318-4 simulator as a cylindrical cavity terminated by an equivalent TI to simulate the IL of earplugs in an ATF ear canal is valid up to $10 \mathrm{kHz}$. Above $6 \mathrm{kHz}$, the simulated IL based on model 2 (LPM - RI) differs significantly from those obtained using the other TI models. This could be supported by the fact that the equivalent TI derived from model 2 (LPM - RI) correlates poorly with that obtained from the others from $6 \mathrm{kHz}$ especially in phase (see Fig.9) in view of the sound propagation in the simulator main cavity poorly described by the LPM. At higher frequencies (over $8.5 \mathrm{kHz}$ ), a mismatch is seen between the IL simulated using model 3 (traditional TI model) and that using model 1 (TM model - RI) (see the "zoomed-in" view of Fig.10). 
This indicates that the adoption of the traditional TI model to calculate the IL of earplugs might be limited to the frequency range below $8.5 \mathrm{kHz}$ if accurate IL simulation is desired. All the IL simulation results in Fig.10 are in satisfactory agreement with the experimental data up to $6 \mathrm{kHz}$. The evident inconsistency between the simulation based on the reference model (FE model of the simulator) and measurements at higher frequencies (above $6 \mathrm{kHz}$ ) is believed to be related to the simplified materiel model of the earplug adopted in the simulation.

\section{Conclusion}

In this work, a TM model of an IEC 60318-4 occluded ear simulator has been proposed based on the geometric information identified from micro-CT scan images. It was established using the LRF model to calculate the impedance of the simulator narrow slits where thermo-viscous energy losses should be taken into account. The TM model was validated using i) a FE model of the scanned simulator in the frequency range [100 Hz, $20 \mathrm{kHz}]$ and ii) impedance measurements with a sound intensity probe $(100 \mathrm{~Hz}-10 \mathrm{kHz})$. The uncertainty propagation of the simulator component dimensions was accounted for using the Monte Carlo method. The TM model was found to better describe the simulator input impedance above $3 \mathrm{kHz}$ compared to the LPM. The equivalent TI was derived from the simulator analytical impedance models (TM model and LPM) using the RI method, and compared to that obtained from the traditional lumped TI model. The various TI models have then been exploited to simulate the IL of a silicone earplug using a FE model of an occluded ATF ear canal (100 Hz - 10 $\mathrm{kHz}$ ). The IL obtained using the TI derived from the TM model (model 1) was found in very good agreement with that acquired using the complete FE model of the simulator (reference model) in the whole frequency range of interest. It confirms that considering the IEC 60318-4 simulator as a cylindrical cavity with a locally reacting terminal impedance condition (equivalent TI) to simulate the IL of earplugs in an ATF ear canal is valid up to $10 \mathrm{kHz}$. In addition, the TM model has been shown to better agree with the simulator FE model than the LPM above $6 \mathrm{kHz}$ regarding the IL simulation since the TI derived from the LPM (model 2) failed to precisely capture the earplug IL in this frequency range. The traditional TI model (model 3) seems adequate when adopted to calculate the earplug IL at frequencies below $8.5 \mathrm{kHz}$ however it becomes less reliable in higher frequency bands. The influence of the studied TI models on the IL simulation was proved to be only pronounced over $6 \mathrm{kHz}$. The TM model of the IEC 60318-4 simulator proposed in this work with acoustic parameters directly related to the simulator geometric dimensions constitute a step forward for designing artificial ear simulators that meet the authors' needs for future investigations.

\section{Acknowledgement}

The authors wish to acknowledge the financial support provided by the Natural Sciences and Engineering Research Council of Canada. 


\section{A Input impedance of the Helmholtz resonators}

\section{A.1 Input impedance of the first Helmholtz resonator}

$$
Z_{H R, 2}=Z_{\text {slit }, 2}+Z_{\text {cav }, 2}
$$

where $Z_{\text {slit,2 }}$ and $Z_{c a v, 2}$ denote the impedance of the rectangular slit and the first side cavity. The former can be derived from:

$$
Z_{s l i t, 2}=j Z_{l, 2} \tan \left(k_{l, 2}\left(a_{2}+2 \triangle l_{2}\right)\right)
$$

where $k_{l, 2}$ and $Z_{l, 2}$ are the complex wavenumber and characteristic impedance in the LRF model of the rectangular slit which depend on the mean values of the corresponding thermal and viscous fields $K_{h, 2}, K_{v, 2}$ and the modified mean thermal field $K_{h, 2}^{\prime}[23]$.

$$
\begin{gathered}
k_{l, 2}^{2}=k_{0}^{2} \frac{K_{h, 2}^{\prime}}{K_{v, 2}}, \quad Z_{l, 2}^{2}=\frac{\left(\rho_{0} c_{0}\right)^{2}}{S_{s l i t, 2}^{2} K_{h, 2}^{\prime} K_{v, 2}} \\
K_{h, 2}^{\prime}=\gamma-(\gamma-1) K_{h, 2} \\
K_{h(v), 2}=1-\frac{\tan \left(k_{h(v)} h_{2} / 2\right)}{k_{h(v)} h_{2} / 2}
\end{gathered}
$$

$k_{h}$ and $k_{v}$ in the equation above denote the thermo-viscous wavenumbers which are associated with the thermal and viscous characteristic lengths $l_{h}=\lambda /\left(\rho_{0} c_{0} C_{p}\right)$ and $l_{v}^{\prime}=\mu /\left(\rho_{0} c_{0}\right)$ (see Table 2 for parameters of the thermoviscous fluid).

$$
k_{h}=\frac{1-j}{\sqrt{2}} \sqrt{k_{0} / l_{h}}, \quad k_{v}=\frac{1-j}{\sqrt{2}} \sqrt{k_{0} / l_{v}^{\prime}}
$$

When calculating the total impedance of the IEC 60318-4 simulator, end corrections should be added to the actual lengths of the narrow slits to account for the radiation impedance at the corresponding locations. Thus, the end correction for a baffled rectangular piston according to [27] is adopted:

$$
\begin{aligned}
& \frac{\triangle l_{2}}{h_{2}}=\frac{1}{3 \pi}\left[\beta_{2}+\frac{1-\varepsilon_{2}^{3 / 2}}{\beta_{2}^{2}}\right]+ \\
& \frac{1}{\pi}\left[\frac{1}{\beta_{2}} \ln \left(\beta_{2}+\sqrt{\varepsilon_{2}}\right)+\ln \left(\frac{1}{\beta_{2}}\left(1+\sqrt{\varepsilon_{2}}\right)\right)\right]
\end{aligned}
$$


where $\varepsilon_{2}=1+\beta_{2}^{2}$ and $\beta_{2}=h_{2} / b_{2}$ is the ratio of the thickness of the narrow slit over the width.

$$
Z_{c a v, 2}=\frac{j \rho_{0} c_{0}\left[B_{s, 2} J_{0}\left(k_{0} r_{2}\right)-Y_{0}\left(k_{0} r_{2}\right)\right]}{S_{c a v, 2}\left[B_{s, 2} J_{1}\left(k_{0} r_{2}\right)-Y_{1}\left(k_{0} r_{2}\right)\right]}
$$

in which $S_{c a v, 2}$ is the cross-section area of the first side cavity [24]. $J_{0}, J_{1}$ and $Y_{0}, Y_{1}$ are Bessel functions of the first and second kind.

$$
B_{s, 2}=\frac{Y_{1}\left(k_{0} R_{2}\right)}{J_{1}\left(k_{0} R_{2}\right)}
$$

\section{A.2 Input impedance of the second Helmholtz resonator}

$$
Z_{H R, 4}=Z_{\text {slit }, 4}+Z_{\text {cav }, 4}
$$

where $Z_{\text {slit, } 4}$ and $Z_{\text {cav }, 4}$ denote the impedance of the annular slit and the second side cavity. $Z_{\text {slit, } 4}$ can be determined by [24]:

$$
\begin{gathered}
Z_{s l i t, 4}=\frac{j Z_{l, 4}\left[A_{s} J_{0}\left(k_{l, 4} R_{0}^{\text {in }}\right)-Y_{0}\left(k_{l, 4} R_{0}^{\text {in }}\right)\right]}{A_{s} J_{1}\left(k_{l, 4} R_{0}^{\text {in }}\right)-Y_{1}\left(k_{l, 4} R_{0}^{\text {in }}\right)} \\
A_{s}=\frac{Y_{0}\left(k_{l, 4} r_{4}^{\text {out }}\right)}{J_{0}\left(k_{l, 4} r_{4}^{\text {out }}\right)}
\end{gathered}
$$

with $R_{0}^{\text {in }}=R_{0}-\triangle l_{4, \text { in }}$ and $r_{4}^{\text {out }}=r_{4}+\triangle l_{4, \text { out }} . \triangle l_{4, \text { in }}$ and $\triangle l_{4, \text { out }}$ denote respectively the end corrections added to the inner and outer radii of the annular slit which depend on its inner and outer perimeters as the equivalent widths (see Eq.(A.7)).

$$
k_{l, 4}^{2}=k_{0}^{2} \frac{K_{h, 4}^{\prime}}{K_{v, 4}}, \quad Z_{l, 4}^{2}=\frac{\left(\rho_{0} c_{0}\right)^{2}}{S_{\text {slit }, 4}^{2} K_{h, 4}^{\prime} K_{v, 4}}
$$

$k_{l, 4}$ and $Z_{l, 4}$ are the LRF wavenumber and impedance in the annular slit related to the corresponding thermal and viscous fields $K_{h, 4}, K_{v, 4}$ and $K_{h, 4}^{\prime}[23]$.

$$
\begin{gathered}
K_{h, 4}^{\prime}=\gamma-(\gamma-1) K_{h, 4} \\
K_{h(v), 4}=1-\frac{\tan \left(k_{h(v)} h_{4} / 2\right)}{k_{h(v)} h_{4} / 2} \\
Z_{c a v, 4}=\frac{j \rho_{0} c_{0}\left[B_{s, 4} J_{0}\left(k_{0} r_{4}\right)-Y_{0}\left(k_{0} r_{4}\right)\right]}{S_{c a v, 4}\left[B_{s, 4} J_{1}\left(k_{0} r_{4}\right)-Y_{1}\left(k_{0} r_{4}\right)\right]}
\end{gathered}
$$


$S_{c a v, 4}$ in Eq.(A.16) is the cross-section area of the second side cavity.

$$
B_{s, 4}=\frac{Y_{1}\left(k_{0} R_{4}\right)}{J_{1}\left(k_{0} R_{4}\right)}
$$

\section{B Glossary}

Table B.1: List of abbreviations

\begin{tabular}{ll}
\hline ATF & Acoustical Test Fixture \\
CT & Computed Tomography \\
FE & Finite Element \\
IL & Insertion Loss \\
LPM & Lumped Parameter Model \\
LRF & Low Reduced Frequency \\
RI & Reduced Impedance \\
TI & Tympanic Impedance \\
TM & Transfer Matrix \\
\hline
\end{tabular}

\section{References}

[1] ANSI/ASA S3.36-2012. American National Standard Specification for a Manikin for simulated in-situ Airborne Acoustic Measurements. Standard, American National Standards Institute, 2012.

[2] ANSI/ASA S12.42-2010. Methods for the measurement of insertion loss of hearing protection devices in continuous or impulsive noise using microphone-in-real-ear or acoustic test fixture procedures. Standard, American National Standards Institute, 2010.

[3] E. H. Berger. Methods of measuring the attenuation of hearing protection devices. The Journal of the Acoustical Society of America, 79(6):1655-1687, 1986.

[4] P. V. Brüel, E. Frederiksen, H. Mathiasen, G. Rasmussen, and E. Sigh. Impedance of real and artificial ears-Part I: Investigations of a new insert earphone coupler. Technical report, 1976.

[5] IEC 60318-4: 2010. Simulators of human head and ear-Part 4: Occluded-ear simulator for the measurement of earphones coupled to the ear by means of ear inserts. Standard, International Electrotechnical Commission, 2010.

[6] ANSI/ASA S3.25-2009. American National Standard for an Occluded Ear Simulator. Standard, American National Standards Institute, 2009. 
[7] S. Jonsson, B. Liu, L. Nielsen, and A. Schuhmacher. Simulation of couplers. AES, Workshop 7, 2003.

[8] L. Nielsen, A. Schuhmacher, B. Liu, and S. Jonsson. Simulation of the IEC 60711 occluded ear simulator. In Audio Engineering Society Convention 116. Audio Engineering Society, 2004.

[9] A. Bravo, R. Barham, M. Ruiz, J. M. López, G. De Arcas, and M. Recuero. A new 3D finite element model of the IEC 60318-1 artificial ear. Metrologia, 45(4):448, 2008.

[10] M. Sasajima, T. Yamaguchi, M. Watanabe, and Y. Koike. FEM Analysis of Occluded Ear Simulator with Narrow Slit Pathway. International Journal of Mechanical, Aerospace, Industrial, Mechatronic and Manufacturing Engineering, 9(9):1430-1433, 2015.

[11] COMSOL Multiphysics 5.3a. Generic 711 Coupler - An Occluded Ear Canal Simulator. https://www. comsol.com/model/generic-711-coupler-8212-an-occluded-ear-canal-simulator-12227, 2017.

[12] D. H. Keefe, R. Ling, and J. C. Bulen. Method to measure acoustic impedance and reflection coefficient. The Journal of the Acoustical Society of America, 91(1):470-485, 1992.

[13] P.-E. Sanborn. Predicting hearing aid response in real ears. The Journal of the Acoustical Society of America, 103(6):3407-3417, 1998.

[14] B. L. Farmer-Fedor and R. D. Rabbitt. Acoustic intensity, impedance and reflection coefficient in the human ear canal. The Journal of the Acoustical Society of America, 112(2):600-620, 2002.

[15] A. Bravo, R. Barham, M. Ruiz, J. M. López, G. De Arcas, and J. Alonso. A new 3D finite element model of the IEC 60318-1 artificial ear: II. Experimental and numerical validation. Metrologia, 49(6):785, 2012.

[16] IEC 60318-1: 1998. Electroacoustics-Simulators of human head and ear-Part 1: Ear Simulator for the Calibration of Supra-aural Earphones. Standard, International Electrotechnical Commission, 1998.

[17] D. Rodrigues, T. Lavergne, E. S. Olsen, T. Fedtke, R. Barham, and J. Durocher. Methodology of designing an occluded ear simulator. Acta Acustica united with Acustica, 101(5):1007-1015, 2015.

[18] G. Viallet, F. Sgard, F. Laville, and J. Boutin. Axisymmetric versus three-dimensional finite element models for predicting the attenuation of earplugs in rigid walled ear canals. The Journal of the Acoustical Society of America, 134(6):4470-4480, 2013.

[19] G. Viallet, F. Sgard, F. Laville, and J. Boutin. A finite element model to predict the sound attenuation of earplugs in an acoustical test fixture. The Journal of the Acoustical Society of America, 136(3):1269-1280, 2014.

[20] A. Chaigne. Ondes acoustiques. Editions Ecole Polytechnique, 2001. pp. 50-52.

[21] H. Hudde. Measurement of the eardrum impedance of human ears. The Journal of the Acoustical Society of America, 73(1):242-247, 1983. 
[22] V. D. Larson, J. A. Nelson, W. A. Cooper, and D. P. Egolf. Measurements of acoustic impedance at the input to the occluded ear canal. Journal of rehabilitation research and development, 30:129-129, 1993.

[23] W. R. Kampinga. Viscothermal acoustics using finite elements-analysis tools for engineers. PhD thesis, 2010 .

[24] D. Rodrigues, C. Guianvarc'h, J.-N. Durocher, M. Bruneau, and A.-M. Bruneau. A method to measure and interpret input impedance of small acoustic components. Journal of Sound and Vibration, 315(4-5):890-910, 2008.

[25] D. T. Blackstock. Fundamentals of physical acoustics. John Wiley \& Sons, 2000. pp. 298-327.

[26] J. Schroeter and C. Poesselt. The use of acoustical test fixtures for the measurement of hearing protector attenuation. Part II: Modeling the external ear, simulating bone conduction, and comparing test fixture and real-ear data. The Journal of the Acoustical Society of America, 80(2):505-527, 1986.

[27] M. L. Munjal, M. Vorländer, P. Költzsch, M. Ochmann, A. Cummings, W. Maysenhölder, and W. Arnold. Formulas of acoustics. Springer Science \& Business Media, 2008. p. 319. 